\title{
Opportunities for enhancing pregnancy planning and preconception health behaviours of
}

\section{Australian women}

Adina Y. Lang ${ }^{\mathrm{a}}$, Cheryce L. Harrison ${ }^{\mathrm{a}}$, Geraldine Barrett ${ }^{\mathrm{b}}$, Jennifer A. Hall ${ }^{\mathrm{b}}$, Lisa J. Moran ${ }^{\mathrm{a}}$, Jacqueline A. Boyle $\mathrm{a}^{\mathrm{a}}{ }^{*}$

${ }^{a}$ Monash Centre for Health Research and Implementation, School of Public Health and Preventive Medicine, Monash University, Ground Floor, 43-51, Kanooka

Grove, Clayton, Victoria, 3186, Australia

${ }^{b}$ Research Department of Reproductive Health, UCL EGA Institute for Women's Health, 74 Huntley Street, London, WC1E 6AU, United Kingdom

*Corresponding author at: Monash Centre for Health Research and Implementation (MCHRI), School of Public Health and Preventive Medicine, Monash University, Monash Medical Centre, Locked Bag 29, Clayton, Victoria, 3168, Australia.

E-mail addresses: adina.lang@ monash.edu (A.Y. Lang), cheryce.harrison@ monash.edu (C.L. Harrison), g.barrett@ucl.ac.uk (G. Barrett), jennifer.hall@ucl.ac.uk (J.A. Hall), lisa.moran@monash.edu (L.J. Moran), jacqueline.boyle@monash.edu (J.A. Boyle). 


\begin{abstract}
Problem and Background: The preconception period provides a significant opportunity to engage women in healthy behaviour change for improved maternal and child health outcomes. However, there is limited research exploring women's pregnancy planning in Australia.
\end{abstract}

\begin{abstract}
Aim: This study investigated associations between pregnancy planning, socio-demographics and preconception health behaviours in Australian women.
\end{abstract}

Methods: A retrospective cross-sectional survey of pregnant women $\geq 18$-years-of-age recruited through a Victorian public maternity service and a national private health insurer.

Results: Overall 317 women ( $30 \pm 4.7$ years) participated (public: $n=225$, private: $n=92)$. Planned pregnancies were reported by $74 \%$ of women and were independently associated with marital status $(\mathrm{AOR}=5.71$ 95\% CI 1.92-17.00, $\mathrm{p}=0.002)$; having $\leq 2$ children $(\mathrm{AOR}=3.75$ 95\%CI 1.28-11.05, $\mathrm{p}=0.016)$; and having private health insurance ( $\mathrm{AOR}=2.5195 \% \mathrm{CI} 1.08-5.81, \mathrm{p}=0.03)$. Overall, women reported preconception: any folic-acid supplementation (59\%), up-to-date cervical screening (68\%), weight management attempts (75\%), accessing information from health professionals (57\%) and immunisation reviews (47\%). Pregnancy planners were more likely to use folic-acid (AOR=17.13 95\%CI 7.67-38.26, $\mathrm{p}<0.001)$, review immunisations ( $\mathrm{AOR}=2.09$ 95\% CI 1.07-4.10, $\mathrm{p}=0.03)$ and access information $(\mathrm{AOR}=3.2495 \% \mathrm{CI} 1.75-6.00, \mathrm{p}<0.001)$ compared to non-planners. Women $<25-$ years-of-age were less likely to access information (AOR=0.38 95\%CI 0.16-0.89, $\mathrm{p}=0.03$ ) and take folic-acid (AOR=0.23 95\%CI 0.09-0.59, $\mathrm{p}=0.002)$ and were more likely to smoke 3 -months preconception $(\mathrm{AOR}=6.6895 \% \mathrm{CI} 1.24-36.12, \mathrm{p}=0.03)$.

Conclusions: Women with planned and unplanned pregnancies reported variable preconception health behaviour uptake and limited healthcare engagement. Opportunities exist to improve awareness 
and healthcare engagement for optimising preconception health and pregnancy planning benefits including collaborative health promotion. Population-based and targeted approaches reaching pregnancy planners and non-planners are required.

MeSH key words: Pregnancy intention, Preconception care, Preventive Medicine, Women's health, Public Health, Pregnancy.

\section{Statement of Significance}

\begin{tabular}{|l|l|}
\hline Problem or Issue & $\begin{array}{l}\text { Optimising healthy behaviours preconception is critical for maternal } \\
\text { and child wellbeing. However, limited research explores Australian } \\
\text { women's pregnancy preparations. }\end{array}$ \\
\hline What is Already Known & $\begin{array}{l}\text { Globally, community awareness of preconception health is low. } \\
\text { Around half of pregnancies in developed countries are unplanned, } \\
\text { leading to poor preconception healthcare engagement, behaviours and } \\
\text { outcomes. }\end{array}$ \\
\hline What this Paper Adds & $\begin{array}{l}\text { A high proportion of women planned their pregnancy and were more } \\
\text { likely to engage with health professionals and recommended } \\
\text { preconception health behaviours (e.g. folic-acid supplementation, } \\
\text { review immunisation). However, health behaviour uptake varied and } \\
\text { was more limited among a significant minority e.g. younger women. } \\
\text { Establishment of Australian preconception health policy, research and } \\
\text { healthcare is critical. }\end{array}$ \\
\hline
\end{tabular}




\section{INTRODUCTION}

The preconception period provides a significant opportunity to engage women in healthy behaviours for improved maternal and child health outcomes (e.g. reduced risk of maternal gestational diabetes, pre-eclampsia, pregnancy loss, low-birth-weight babies, neonatal mortality and child future chronic disease).${ }^{1,2}$ Guidelines ${ }^{3-6}$ recommend optimising preconception health $(\mathrm{PCH})$ behaviours such as: maintaining nutrition and physical activity; achieving a healthy weight; smoking, drug and alcohol cessation; chronic disease management, immunisations and screening.

Community awareness of the importance of $\mathrm{PCH}$ and its benefits is low, with most healthcare engagement commencing during antenatal care. ${ }^{1}$ Few women seek PCH advice and have limited pregnancy intention discussions with healthcare providers. ${ }^{7,8}$ Preconception care is also underutilised by healthcare providers who report constraints of time and resources, competing priorities and challenges identifying women planning pregnancy. ${ }^{7-10}$

Previous Australian research indicates $\sim 70 \%$ of pregnancies are planned, however estimates are limited by the application of different tools which lack validation in the Australian context ${ }^{11,12}$ and women included were predominantly of a high socio-economic status. ${ }^{11}$ Unplanned pregnancies are associated with poor reproductive healthcare engagement, low PCH behaviour uptake and adverse outcomes globally (e.g. pregnancy loss, low-birth-weight and neonatal mortality). ${ }^{1,2}$ Despite the pragmatic attraction of pregnancy planning and healthy behaviour change preconception, a paucity of evidence explores women's pregnancy planning and preparation behaviours in Australia.

This current study aimed to inform the development of effective approaches to promote PCH by surveying a cross-section of pregnant women in Australia receiving maternity care through both the public and private health systems to (a) investigate pregnancy planning and PCH behaviours and (b) explore associations between pregnancy planning, socio-demographic factors and $\mathrm{PCH}$ behaviours. 


\section{METHODS}

\subsection{Study design}

A retrospective cross-sectional survey of Australian pregnant women assessing pregnancy planning and PCH behaviours.

\subsection{Recruitment, settings and participants}

A sample of reproductive-aged women was recruited through two settings including one of the largest public maternity settings in Australia (Monash Health) and through a large private health insurer [Medibank Private Limited (Medibank)], reflecting the current proportion of public (74\%) and private (26\%) Australian hospital births. ${ }^{13}$

In the Monash Health cohort, 314 eligible women expressed interest and were recruited. Twohundred-and-twenty-five women (71\%) completed the questionnaire. The Medibank membership database identified 4,870 pregnant and non-pregnant women who were invited to participate. Ten percent of women recruited in the Medibank cohort $(n=504)$ completed the questionnaire and only women who identified as being pregnant $(n=92)$ were included in this study (the study flow diagram is reported in a related manuscript by Lang et al. $2019^{14}$ ).

\subsubsection{Monash Health - public maternity service}

Women were recruited at their first clinical antenatal appointment through Monash Health, a large, diverse maternity service, comprising $62 \%$ overseas-born women.

Recruitment processes were established with the maternity service. Invitations were mailed to all women booked for the Monash Health clinic (August 2017-March 2018). Women expressed interest or were recruited face-to-face at their first antenatal visit. Pregnant women over 18-years-of-age, able to speak and write in English were eligible. 
Eligible women completed a secure online or hard copy questionnaire. Reminders were sent as required. In recognition of participation, women were placed in the draw for a \$100AUD gift card.

\subsubsection{Medibank - private health system}

Medibank, a major national Australian private healthcare insurers, co-developed and distributed an email inviting eligible members to participate (women 18-40-years-of-age, who held coverage for obstetrics 12-months before recruitment [January 2018] and self-identified as pregnant). Women opted-in by completing the online questionnaire.

\subsection{Ethics}

Monash Health (RES-17-0000-087A) and Monash University (Project no.10370) Human Research Ethics Committees provided ethics approval. All participants provided informed consent.

\subsection{Measures}

The questionnaire was adapted from existing tools and aligned with The Royal Australian College of General Practitioners, Guidelines for preventive activities in general practice ${ }^{3}$ to assess women's pregnancy planning and preparations. ${ }^{3,4,15-17}$ A questionnaire developed by Stephenson et al ${ }^{15}$ formed the basis for the questions, topics and themes. To ensure all relevant key topics were included and to assist with question phrasing, assessment was also made against a US preconception screening checklist ${ }^{17}$ and the nine key evidence based preconception wellness measures agreed upon by the Clinical workgroup of the National Preconception Health and Health Care Initiative ${ }^{4}$. The questionnaire underwent an extensive process of development and review by the multidisciplinary research team with expertise in women's health, maternal and child health, exercise physiology, dietetics and endocrinology. The final questionnaire included 59 questions and covered: circumstances, feelings and planning around pregnancy (using the London Measure of Unplanned Pregnancy, LMUP, described below); health behaviours and lifestyle information; PCH information received; medical history and previous pregnancies; and socio-demographic information. 


\subsubsection{Pregnancy planning}

The questionnaire included the six-question London Measure of Unplanned Pregnancy (LMUP), ${ }^{16}$ which was validated with this cohort to measure pregnancy planning in Australian women (http://measure.ascody.co.uk/lmupaustralia.htm). ${ }^{14}$ Findings of the validation study showed this was a valid, reliable and internally consistent measure with excellent stability (alpha $=0.81$, corrected itemtotal correlations all $>0.20$, inter-item correlations all positive, weighted kappa $=0.92) .{ }^{14}$ The LMUP offers a comprehensive, sensitive measure accounting for the nuanced complexities, feelings and behaviours surrounding conception. ${ }^{16}$ Questions are scored and summed producing a total score $(0-$ 12), with higher scores indicating higher planning levels. ${ }^{18}$ Scores $\geq 10$ were classified as planned and $0-9$, unplanned. ${ }^{18}$

The questionnaire (including the embedded LMUP) was tested using cognitive interviews, ${ }^{19}$ ensuring comprehension and suitability. Cognitive interviews were conducted with six pregnant women recruited through the public maternity service only as it was not possible to conduct follow-up interviews through the private health system due to anonymity of the participants. Women did not report any issues with the comprehension of the questionnaire and only minor changes were made to some of the questionnaire instructions and question phrasing based on a further review of the questionnaire by the research team. Further detail of the LMUP validation study, cognitive interview methods and participants are reported in Lang et al. 2019. ${ }^{14}$

\subsubsection{Socio-demographic characteristics}

Women recruited through Medibank self-reported their socio-demographic characteristics within the study questionnaire only (including age, relationship status, place of birth, previous live birth, number of children, education, employment and postcode, Table 1). Women recruited through Monash Health self-reported their age, relationship status, place of birth, education and employment within the study questionnaire. Additional information was obtained from Monash Health medical records for: gestation at first antenatal visit (when recruitment occurred), previous live births, number of children 
and postcode. Data on gestation at recruitment through the Medibank was not collected.

Socioeconomic status was determined based on the alignment of participant postcodes with corresponding deciles from the Australian Socio-economic Indexes for Areas (SEIFA), Index of Relative Socio-economic Disadvantage. ${ }^{20}$ Deciles 1-5 indicate higher level disadvantage and decile 610, lower level of disadvantage. Additional details on the measurement of socio-demographic information have been reported in the Australian LMUP validation manuscript, see Lang et al. 2019. ${ }^{14}$

\subsubsection{Medical history and PCH behaviours}

Information was gathered within the study questionnaire on pre-existing medical conditions and medication use in the 6-months before pregnancy. ${ }^{15}$ This included medical conditions (e.g. anxiety, depression, asthma, thyroid disease, polycystic ovary syndrome, anaemia) or teratogenic medications (e.g. to treat asthma, anxiety, depression, epilepsy, thyroid disease) that have been identified within the literature as potentially causing adverse effects on pregnancy and for which medical review is advised in the 6-months prior to pregnancy. ${ }^{3,15}$ Women indicated whether they took preconception folic-acid supplementation (alone/multivitamins at any time preconception) as part of the LMUP, and were asked whether they had undertaken cervical screening in the previous 2-years and dental visits 12-months preconception as part of the broader questionnaire. Responses were categorised Yes/No. Immunisation status review 12-months preconception was asked in the questionnaire, with responses classified Yes if women selected that their immunisation status for Measles, Mumps, Rubella (MMR) and/or, Hepatitis B and/or Chickenpox had been checked within the 12-months prior to conception.

The questionnaire asked women "Did you attempt to maintain a healthy weight or lose weight in the 6-months before you became pregnant?', response options were: 'Yes, maintain a healthy weight', 'Yes, lose weight' or 'No'. These weight management intentions were combined and classified Yes (lose or maintain weight)/No. Women reported ever smoking, drinking alcohol or taking illicit drugs and whether these behaviours continued 3-months preconception. Information accessed from a 
general practitioner/other health professional (at any time point preconception) on any PCH topic was asked with responses classified Yes/No.

\subsection{Statistical analysis}

Descriptive statistics are presented as median (inter-quartile range [IQR]), frequencies (percentages), for continuous and categorical data respectively. Gestation was unavailable for Medibank participants. The Mann-Whitney U and Chi-square test were used to compare medians and frequencies (percentages) of maternal characteristics and behaviours of women with planned or unplanned pregnancies. Univariable and multivariable logistic regression assessed associations. Multivariable models included covariates yielding $\mathrm{p}$-values $<0.1$ or that were determined clinically significant a priori. Data were analysed using IBM SPSS Statistics version 25 (Armonk, New York). The level of significance was set at $5 \%$.

\section{RESULTS}

\subsection{Participants}

The questionnaire was completed by 317 pregnant women from the public $(n=225)$ and private $(n=92)$ maternity care settings.

\subsection{Characteristics of women}

Median (IQR) age overall and gestation of women attending public maternity services was $30(27,33)$ years and $7(5.5,11.0)$ weeks pregnant at recruitment. Most women were married/de facto, attained post-secondary school education and were in paid employment one-year preconception.

Approximately half of women had previous live births and were non-Australian-born. Nine-percent of women reported receiving fertility treatment for their current pregnancy. Participant sociodemographics (Table 1) were similar to national averages for pregnant women for age (average age 30 years in the study cohort vs 30.5 years nationally), place of birth ( $42.5 \%$ of women born outside 
Australia in the current study vs $34.4 \%$ nationally), previous live birth (51.6\% in this study vs $56.8 \%$ nationally) and public/private healthcare (71\% public hospital births in this study vs $74 \%$ nationally). The exception was marital status where $93 \%$ of women in the study were married/de facto, compared to $66 \%$ of all women who give birth in Australia. ${ }^{13,21}$

\subsection{Pregnancy planning}

Seventy-four percent $(n=236 / 317)$ of women planned their pregnancies (LMUP score 10-12) and $26.6 \%(\mathrm{n}=81 / 317)$ were unplanned (LMUP score $0-9)$.

\subsection{Characteristics of women and pregnancy planning}

Table 2 presents relationships between maternal characteristics and pregnancy planning. On multivariable analysis adjusting for socio-demographics, pregnancy planning was significantly associated with being married/de facto ( $\mathrm{AOR}=5.7195 \% \mathrm{CI} 1.92-17.00, \mathrm{p}=0.002)$; having $\leq 2$ children, $(\mathrm{AOR}=3.7595 \% \mathrm{CI} 1.28-11.05, \mathrm{p}=0.02)$; and having private health insurance $(\mathrm{AOR}=2.5195 \% \mathrm{CI}$ $1.08-5.81, \mathrm{p}=0.03)$.

\subsection{PCH behaviours}

Among all women, $74.8 \%(n=228 / 305)$ reported preconception weight management, $67.5 \%$ $(\mathrm{n}=185 / 274)$ up-to-date cervical screening, $58.7 \%(\mathrm{n}=186 / 317)$ any folic-acid use, 56.6\% $(\mathrm{n}=162 / 286)$ accessing preconception information from health professionals, 55.8\% ( $\mathrm{n}=168 / 301)$ visiting a dentist and $47.0 \%(n=118 / 286)$ reviewed immunisations (Table 1$)$.

Over one-third of women $(n=30 / 87)$ continued smoking, $64.4 \%(n=139 / 216)$ continued to drink $\geq 1$ standard alcoholic drink per week (Table 1) and five women (9.6\% of the 52 women who reported ever using illicit drugs) continued to use illicit drugs 3-months preconception. 


\subsubsection{Supplementation (iodine and vitamin D)}

Overall 64 women $(20 \%)$ reported taking iodine $(n=35 / 225,15.5 \%$ public and $n=29 / 92,31.5 \%$ private participants). Preconception vitamin D supplementation alone was reported by $34.7 \%(n=110 / 317)$ of women overall ( $n=79 / 225,35.1 \%$ public and $n=31 / 92,33.7 \%$ private participants). Data available for public participants indicated $29.8 \%$ of women $(\mathrm{n}=67 / 225)$ had low vitamin $\mathrm{D}$ levels $<50 \mathrm{ng} / \mathrm{mL}$, $34.3 \%(n=23 / 67)$ of which reported supplementation.

\subsubsection{Relevant medical condition, medications and health professional engagement}

Half of women $(n=134 / 274)$ reported relevant medical conditions (i.e. medical conditions that have potentially adverse effects on pregnancy and require a medical review 6-months before pregnancy) 6months preconception (e.g. anxiety, depression, epilepsy, thyroid disease, polycystic ovary syndrome), of which $69.4 \%(n=93 / 134)$ saw health professionals about their condition and $43.0 \%$ $(n=40 / 93)$ of these health professionals asked about these women's pregnancy plans.

Overall, $15.1 \%(\mathrm{n}=48 / 317)$ of women reported taking teratogenic medications 6-months preconception. Almost three-quarters of these women $(n=34 / 48)$ reported their medication was medically reviewed and discussed with their doctor in preparation for pregnancy.

\subsection{Pregnancy planning, socio-demographics and health behaviours}

Multivariable analysis showed that women planning pregnancy had significantly greater odds of: any folic-acid use (AOR=17.13 95\%CI 7.67-38.26, p<0.001); reviewing immunisations (AOR=2.09 95\%CI 1.07-4.10, $\mathrm{p}=0.03)$; and accessing information from health professionals $(\mathrm{AOR}=3.2495 \% \mathrm{CI}$ 1.75-6.00, $\mathrm{p}<0.001$; Table 3). Women planning pregnancy had significantly lower odds of: drinking alcohol 3-months preconception (AOR=0.34 95\%CI 0.16-0.70, $\mathrm{p}=0.004)$ and ever taking illicit drugs $(\mathrm{AOR}=0.4495 \% \mathrm{CI} 0.21-0.90, \mathrm{p}=0.03)$. Smoking was negatively associated with pregnancy planning on univariable but not multivariable analysis. 


\subsubsection{Other significant socio-demographic predictors of health behaviours}

Multivariable analysis showed that women with private health insurance had significantly greater odds of dental visits (AOR=2.28 95\%CI 1.17-4.43, $\mathrm{p}=0.02)$, ever use of illicit drugs (AOR=3.63 95\% CI 1.68-7.82, $\mathrm{p}=0.001)$ and continuing to drink alcohol ( $\geq 1$ standard drink/week) 3-months preconception $(\mathrm{AOR}=3.55$ 95\%CI 1.64-7.71, $\mathrm{p}=0.001)$.

Women $<25$-years-of-age had significantly lower odds of accessing information from health professionals $(\mathrm{AOR}=0.38$ 95\% CI 0.16-0.89, $\mathrm{p}=0.03)$, taking folic-acid $(\mathrm{AOR}=0.23$ 95\%CI 0.09-0.59, $\mathrm{p}=0.002)$ and up-to-date cervical screening $(\mathrm{AOR}=0.23$ 95\% CI 0.10-0.53, $\mathrm{p}=0.001)$, with greater odds of continuing smoking 3-months preconception (AOR=6.68 95\%CI 1.24-36.12, $\mathrm{p}=0.03)$. Women with post-secondary school education had significantly reduced odds of ever using illicit drugs $(\mathrm{AOR}=0.39$ 95\% CI 0.17-0.91, $\mathrm{p}=0.03)$.

Australian-born women had significantly greater odds of up-to-date cervical screening compared to non-Australian-born women $(\mathrm{AOR}=2.6495 \% \mathrm{CI} 1.47-4.74, \mathrm{p}=0.001)$. Women with children had significantly lower odds of reviewing immunisations than women without children (AOR $=0.57$ 95\%CI 0.33-0.99, $\mathrm{p}=0.05)$.

\section{DISCUSSION}

To our knowledge, this is the first Australian study investigating associations between a comprehensive range of PCH behaviours, pregnancy planning and socio-demographic characteristics among women receiving maternity care in both public and private health systems. In this cohort, we report high pregnancy planning rates $(74 \%)$. Women were more likely to plan for their pregnancy if they were married/de facto, had $\leq 2$ children or had private health insurance and those who planned their pregnancy were more likely to follow $\mathrm{PCH}$ recommendations. However, rates of risk-taking behaviours (e.g. $34.5 \%$ of women who had ever smoked continued smoking and $64.4 \%$ of women 
who had ever drank alcohol continued drinking) remained concerning among both women planning and not planning pregnancy. Younger women were less likely to access information from health professionals or use folic-acid and were more likely to smoke during the three month preconception period. Overseas-born women were less likely to have up-to-date cervical screening. Health professional engagement for preconception information was limited overall, and women with preexisting illness were not routinely being asked about their pregnancy intentions by their doctors.

Similar to prior studies in antenatal settings, planned pregnancies were highly prevalent among our cohort. ${ }^{11,15}$ Women with planned pregnancies had greater odds of being married/de facto, having $\leq 2$ children and having private health insurance compared to women with unplanned pregnancies. Women with $\geq 3$ children may hold higher degrees of complacency in planning subsequent pregnancies. ${ }^{22}$ Similarly, women with children were less likely to report reviewing their immunisation status than those without children, possibly owing to review and subsequent awareness in their previous pregnancy. Consistent with prior research, women who were married/de facto reported higher levels of planning ${ }^{11}$ perhaps due to social norms and expectations of married women having children (particularly in some cultures), financial and relationship stability and having partner support among other factors. ${ }^{23,24}$ Those with private health insurance have been found to be more engaged in pregnancy planning and $\mathrm{PCH}$ behaviours, possibly due to their financial investment in private healthcare for pregnancy. ${ }^{12,25}$ Pregnancy planning was broadly associated with greater uptake of most recommended PCH behaviours. However, the uptake of health behaviour change was varied (e.g. from $53 \%$ of women reviewing their immunisations and $56 \%$ having a prior dental visit, to $75 \%$ of women taking folic-acid and $76 \%$ trying to lose or maintain their weight). This variation is potentially related to a range of factors such as differences in women's PCH awareness, past pregnancy experiences, perceptions regarding the relevance of different behaviours to women's current lifestyles and the complexities surrounding behaviour change. ${ }^{1}$ These findings emphasise the need to explore opportunities and interventions for improvement, particularly capitalising on engaging women 
planning pregnancies who may have limited knowledge and supports to enable appropriate behaviour change.

A significant proportion of women continued to smoke and drink alcohol in the preconception period, particularly women with unplanned pregnancies. Interestingly, holding private health insurance significantly increased the odds of continuing alcohol consumption 3-months preconception or ever taking illicit drugs. Similarly, Australian research has reported $60 \%$ of women consume alcohol prior to pregnancy awareness, with higher rates among women from higher socio-economic background or who have reported unplanned pregnancies. ${ }^{26}$ This may reflect Australian drinking culture, low awareness, conflicting and unclear messaging regarding safe levels of preconception alcohol consumption and lack of accessible preconception resources and services. ${ }^{7,27}$ While Australian guidelines recommend alcohol abstinence in preconception and pregnancy, with warning labels and complimentary pregnancy alcohol cessation initiatives, high preconception alcohol consumption remains a concern. ${ }^{27}$

Contrary to other Australian findings ${ }^{11}$ women's age was not related to pregnancy planning but was pertinent to women's health behaviours. Women <25-years-of-age reported a lower likelihood of accessing information from health professionals or taking folic-acid, and higher likelihood of smoking 3-months preconception, compared to women $\geq 25$-years-of-age. Research suggests younger women may be less aware of optimising pre-pregnancy health, relating the risks to pregnancy rather than preconception. ${ }^{28}$ This suggests different approaches need to be investigated to reach young women. For example, literature suggests younger women could benefit from overall healthy lifestyle programs within schools, community health settings and through marketing, social media and mobile phone apps. ${ }^{29,30}$

Here we report that pregnancy planning rates were similar across women regardless of their country of birth as were most health behaviours apart from overseas-born women being less likely to report an 
up-to-date cervical screening. Australian findings regarding influences of country of birth on pregnancy planning are mixed. Rassi et al reported women of Asian background were more likely to report unintended pregnancies compared to other ethnicities, a factor which was not explored in the current study. ${ }^{11}$ Conversely, Rowe et al found no difference among women but showed overseas-born men were more likely than Australian-born men to be partners in unplanned pregnancies. ${ }^{12}$ However, it is important to note that Rowe et al used a single question to assess pregnancy intention within their study asking "How many times that you know about have you, or you together with a partner, had an accidental pregnancy?" and explored pregnancy planning among both women and men. The similarity in the uptake of $\mathrm{PCH}$ behaviours present among Australian and overseas-born women in this study may be reflective of the cohort being English-speaking and predominantly of a higher socio-economic status. Cultural sensitivities, inappropriate resources or women having no screening practices in their home country, however, might explain differences in cervical screening behaviours. ${ }^{31}$ Given that a quarter ${ }^{13}$ of women giving birth in Australia come from non-English speaking countries and their cultural beliefs, practices and experiences often influence their pregnancy preparations,${ }^{24}$ culturally responsive health promotion and healthcare approaches are imperative.

Just over half of women in our study reported health professional engagement during pregnancy preparation. This is consistent with previous reports of women's low awareness of, and presentation for preconception care. ${ }^{7,8}$ Whilst many women with pre-existing medical conditions reported visiting health professionals preconception, only $43 \%$ reported health professionals queried their pregnancy intentions, despite the fact that the clinical guidelines recommend general practitioners ask clients about their reproductive life plans. ${ }^{3}$ Encouragingly, however, most women taking teratogenic medications reported a doctor's review. Findings point to much needed improved engagement of women but also training and support for health professionals who report difficulties identifying women planning pregnancies, having limited time, resources and many competing priorities. ${ }^{7,9,10}$ While women may not regularly visit their general practitioner, they may be more likely to see other health professionals. Prior research and international healthcare models suggest other health 
professionals who see women before or between pregnancies (e.g. midwives and maternal and child health nurses) could play a role in the continuum of preconception care, particularly between pregnancies. ${ }^{32-35}$ Consideration of promising innovative digital solutions such as interactive conversational agents as an adjunct to face-to-face care could also be trialled to overcome some of the noted barriers in the delivery of preconception care. ${ }^{36}$ Given our findings, further exploration of these models of care and extending the scope of practice for these professionals is warranted.

Discussions implemented within routine lifestyle checks and opportunistically during sexual and reproductive healthcare merit investigation. ${ }^{37}$ Reproductive life planning ${ }^{37}$ approaches by healthcare providers have been advocated internationally as a means to enable a structured opportunity to address life planning, reproductive desires and initiate PCH discussions and care. While implementation research and evidence for the benefits of reproductive life planning is limited, Morse and Moos suggest reproductive life planning has the potential to increase empowerment and agency, influence pregnancy intendedness, align contraceptive choices with reproductive goals and raise understanding of the importance of optimising $\mathrm{PCH} .{ }^{37,38} \mathrm{It}$ is important that evaluation of the feasibility and effectiveness of such approaches take into account models of care addressing the constraints identified by primary care providers. ${ }^{8,9,32-35}$

\subsection{Implications for policy and practice}

Together with international policy and research, ${ }^{39}$ these findings support the need for collaborative multi-strategic action by policy-makers, health professionals, researchers and consumers to increase the implementation of approaches to improve uptake of recommended PCH behaviours and engagement with healthcare providers. This requires system-wide approaches to $\mathrm{PCH}$ promotion across the healthcare continuum (health promotion, healthcare and policy) and life-course, reaching all reproductive-aged women and those with immediate pregnancy intentions. Multiple strategies could include broad awareness-raising campaigns across diverse settings (e.g. schools, primary care, workplaces, childcare, maternal and child services and through private health insurers) and strategies 
(e.g. social marketing, garnering social movement and signposting evidence-based online resources). ${ }^{30}$ Health professional training, support and adaptive models of care are required to address barriers to PCH consultations as well as exploring adjunct digital technologies ${ }^{36}$ and extending the scope of health professionals who could be involved in preconception care more broadly. ${ }^{7,9,}$ 10, 32-35 Additionally exploration of innovations to engage non-planners is critical, as well as targeted interventions which may benefit some women such as those who are younger, from culturally and linguistically diverse backgrounds or with pre-existing medical conditions. Inclusion of the validated Australian LMUP as part of routine information collected through maternity services could be investigated to support $\mathrm{PCH}$ surveillance and intervention monitoring and evaluation, an approach which is currently being piloted in the United Kingdom. ${ }^{40}$

\subsection{Strengths and limitations}

Our study provides insights into Australian women's PCH behaviours. Public and private healthcare recruitment captured a broad cross-section of Australian women, including $42.5 \%$ of women born overseas from culturally and linguistically diverse backgrounds. While the cohort was generally similar to the national population of pregnant women, much higher rates of women in this study reported being married/de facto. The study may overestimate the uptake of the preconception behaviours examined given higher rates of pregnancy planning in married/de facto women. However, on multivariable analysis being married/de facto was not independently associated with any of the health behaviours examined. While women were recruited through a Victorian hospital and national private health insurer with representation of at least one woman from every state in Australia, the majority of women (87\% of those with an available postcode) were from the state of Victoria, and the sample may not be representative of all Australian women. Participant response rates were precluded due to anonymity in the private health system and inability to screen all women visiting the public maternity hospital. The retrospective design in an antenatal population may have missed terminated pregnancies. However, data collection was early in pregnancy. While overseas-born women were represented in the study, only English-speaking women participated. Though most participating 
women lived in areas of lower levels of socio-economic disadvantage, there was $30 \%$ representation from women living in areas with a high risk of disadvantage. This study did not measure consistency of engagement for all PCH behaviours and in some cases (such as folic-acid use and accessing health information), "any" preconception engagement was included. Future research should provide quantification and timelines for engagement in PCH behaviours. Capturing of women's weight management intentions was limited as the question response options did not allow any further insight into women's feelings towards weight maintenance or management. Exploration of men's PCH including examination of how men's demographic factors predict their partner's PCH is also important for future research.

\section{CONCLUSION}

$\mathrm{PCH}$ is recognised as vital for optimising the health of women, their pregnancies and their subsequent children. Here we report most women planning pregnancy, yet there was variable uptake of recommended PCH behaviours and limited engagement with healthcare providers. Additionally, healthcare providers were not talking to women about their pregnancy intentions. This suggests opportunities to engage with women planning pregnancies in addition to raising general community awareness of optimising preconception and reproductive health and benefits of pregnancy planning. 


\section{Ethical statement}

Ethics approval for this study was granted on 20 July 2017 by the Monash Health (RES-17-0000087A) and Monash University (Project no. 10370) Human Research Ethics Committees.

\section{Author statement}

A.Y.L. conceived, designed and implemented the study and performed the data collection, analysis and interpretation under the supervision of J.A.B.; C.L.H. contributed to conception, design and interpretation; A.Y.L. fully drafted and prepared the original manuscript and revisions and all authors (A.Y.L., C.L.H., G.B., J.A.H., L.J.M., J.A.B.) critically reviewed and approved the final version of the manuscript.

\section{Conflict of interest}

None declared.

\section{Acknowledgements}

We acknowledge Monash Health and Medibank Private Limited and all clinic midwives, staff and participants for supporting this study. Medibank Private Limited provided funding and in-kind support to enable study recruitment, which would not have otherwise been possible. We thank Mr Sanjeeva Ranasinhaat at Monash Centre for Health Research and Implementation for statistical analysis advice. This research received no external funding. Adina Lang and Jacqueline Boyle are supported by a National Health and Medical Research Council Postgraduate Research Scholarship and Career Development Fellowship respectively. Lisa Moran is supported by a National Heart Foundation Future Leader Fellowship. 


\section{REFERENCES}

1. Stephenson J, Heslehurst N, Hall J, Schoenaker DAJM, Hutchinson J, Cade JE, et al. Before the beginning: nutrition and lifestyle in the preconception period and its importance for future health. Lancet. 2018;391(10132):1830-41.

2. Hall JA, Benton L, Copas A, Stephenson J. Pregnancy intention and pregnancy outcome: systematic review and meta-analysis. Matern Child Health J. 2017; 21(3): 670-704. 3. RACGP. Guidelines for preventive activities in general practice. 9th edn East Melbourne: Vic: RACGP; 2016 [cited 2019 May 8]. Available from: http://www.racgp.org.au/your-practice/guidelines/redbook/.

4. Frayne DJ, Verbiest S, Chelmow D, Clarke H, Dunlop A, Hosmer J, et al. Health care system measures to advance preconception wellness: consensus recommendations of the clinical workgroup of the national preconception health and health care initiative. Obstet Gynecol. 2016; 127(5): 863-72.

5. WHO. Meeting to develop a global consensus on preconception care to reduce maternal and childhood mortality and morbidity. Geneva: WHO; 2013.

6. Women's Health Committee - RANZCOG. Pre-pregnancy counselling 2017 [cited 2019 March 11]. Available from:

https://ranzcog.edu.au/RANZCOG_SITE/media/RANZCOG-

MEDIA/Women\%27s\%20Health/Statement\%20and\%20guidelines/Clinical-Obstetrics/Prepregnancy-Counselling-(C-Obs-3a)-review-July-2017_1.pdf?ext=.pdf.

7. Mazza D, Chapman A, Michie S. Barriers to the implementation of preconception care guidelines as perceived by general practitioners: a qualitative study. BMC Health Serv. Res. 2013;13:36.

8. Mazza D, Chapman A. Improving the uptake of preconception care and periconceptional folate supplementation: what do women think? BMC Public Health. 2010;10:786. 
9. Bateson DJ, Black KI. Pre-conception care: an important yet underutilised preventive care strategy. Med J Aust. 2018; 209(9): 389-91.

10. Kizirian NV, Black KI, Musgrave L, Hespe C, Gordon A. Understanding and provision of preconception care by general practitioners. Aust N Z J Obstet Gynaecol. 2019. Epub 2019 Feb 17. doi: 10.1111/ajo.12962.

11. Rassi A, Wattimena J, Black K. Pregnancy intention in an urban Australian antenatal population. Aust N Z J Public Health. 2013; 37(6): 568-73.

12. Rowe H, Holton S, Kirkman M, Bayly C, Jordan L, McNamee K, et al. Prevalence and distribution of unintended pregnancy: the Understanding Fertility Management in Australia National Survey. Aust N Z J Public Health. 2016;40(2):104-9.

13. AIHW. Australia's mothers and babies 2016 - in brief. 2018. [cited 2018 September 9]. Available from: https://www.aihw.gov.au/reports/mothers-babies/australias-mothersbabies-2016-in-brief/data.

14. Lang AY, Hall JA, Boyle JA, Harrison CL, Teede H, Moran LJ, et al. Validation of the London Measure of Unplanned Pregnancy among pregnant Australian women. PLoS One. 2019;14(8):e0220774

15. Stephenson J, Patel D, Barrett G, Howden B, Copas A, Ojukwu O, et al. How do women prepare for pregnancy? Preconception experiences of women attending antenatal services and views of health professionals. PLoS One. 2014;9(7):e103085.

16. Barrett G, Smith SC, Wellings K. Conceptualisation, development, and evaluation of a measure of unplanned pregnancy. J Epidemiol Community Health. 2004; 58(5): 426-33.

17. State of Illinois - Illinois Department of Healthcare and Family Services.

Preconception screening checklist State of Illinois. Illinois: HFS, 2012. [cited 2019 April 10]. Available from: https://www.illinois.gov/hfs/SiteCollectionDocuments/hfs27pf.pdf.

18. Hall JA, Barrett G, Copas A, Stephenson J. London Measure of Unplanned Pregnancy: guidance for its use as an outcome measure. Patient Related Outcome Measures. 2017;8:43-56. 
19. Willis GB. Cognitive interviewing: a tool for improving questionnaire design.

Thousand Oaks, California: Sage, 2005.

20. ABS. 2033.0.55.001 - Census of population and housing: socio-economic indexes for areas (SEIFA), Australia, 2016. 2018. [cited 2018 July 15] Available from:

http://www.abs.gov.au/AUSSTATS/abs@.nsf/DetailsPage/2033.0.55.0012016?OpenDocume

nt.

21. ABS. 3301.0 - Births, Australia, 2016. 2017. [cited 2018 Aug 16]. Available from: http://www.abs.gov.au/AUSSTATS/abs@.nsf/Latestproducts/3301.0Main\%20Features32016 ?opendocument $\&$ tabname $=$ Summary $\&$ prodno $=3301.0 \&$ issue $=2016 \&$ num $=\& v i e w=$.

22. Goossens J, Beeckman D, Van Hecke A, Delbaere I, Verhaeghe S. Preconception lifestyle changes in women with planned pregnancies. Midwifery. 2018; 56: 112-120.

23. Hewitt B, England P, Baxter J, Shafer EF. Education and unintended pregnancies in Australia: do differences in relationship status and age at birth explain the education gradient? Popul Rev. 2010;49(1):36-52.

24. Lang AY, Bartlett R, Robinson T, Boyle JA. Perspectives on preconception health among migrant women in Australia: A qualitative study. Women Birth. 2019; In press. doi: https://doi.org/10.1016/j.wombi.2019.06.015.

25. Hawks R, McGinn A, Bernstein P, Tobin J. Exploring preconception care: insurance status, race/ethnicity, and health in the pre-pregnancy period. Matern Child Health J. 2018;22(8):1103-10.

26. McCormack C, Hutchinson D, Burns L, Wilson J, Elliott E, Allsop S, et al. Prenatal alcohol consumption between conception and recognition of pregnancy. Alcoholism. 2017;41(2):369-78.

27. Food Regulation Standing Committee. Food regulation standing committee decision regulation impact statement: pregnancy warning labels on packaged alcoholic beverages 2018. [cited 2019 May 8]. Available from: 
http://ris.pmc.gov.au/sites/default/files/posts/2018/10/pregnancy_warning_labels_on_package d_alcoholic_beverages_decision_ris.pdf.

28. Tuomainen H, Cross-Bardell L, Bhoday M, Qureshi N, Kai J. Opportunities and challenges for enhancing preconception health in primary care: qualitative study with women from ethnically diverse communities. BMJ Open. 2013;3(7).

29. Goodfellow A, Frank J, McAteer J, Rankin J. Improving preconception health and care: a situation analysis. BMC Health Serv Res. 2017;17(1):595.

30. Barker M, Dombrowski SU, Colbourn T, Fall CHD, Kriznik NM, Lawrence WT, et al. Intervention strategies to improve nutrition and health behaviours before conception. Lancet. 2018;391(10132):1853-64.

31. Cullerton K, Gallegos D, Ashley E, Do H, Voloschenko A, Fleming M, et al. Cancer screening education: can it change knowledge and attitudes among culturally and linguistically diverse communities in Queensland, Australia? Health Promot J Austr. 2016;27(2):140-7.

32. Hammarberg K, Taylor L. Survey of Maternal, Child and Family Health Nurses' attitudes and practice relating to preconception health promotion. Aust J Prim Health. 2019;25(1):43-48.

33. Braspenningx S, Haagdorens M, Blaumeiser B, Jacquemyn Y, Mortier G. Preconceptional care: a systematic review of the current situation and recommendations for the future. Facts Views Vis Obgyn. 2013;5(1):13-25.

34. Allan HT, Mounce G, Crespo E, Shawe J. Preconception care for infertile couples: nurses' and midwives' roles in promoting better maternal and birth outcomes. J Clin Nurs. 2018;27(23-24):4411-8.

35. M'Hamdi H I, van Voorst SF, Pinxten W, Hilhorst MT, Steegers EA. Barriers in the uptake and delivery of preconception care: exploring the views of care providers. Matern. Child Health J. 2017;21(1):21-8. 
36. Jack B, Bickmore T, Hempstead M, Yinusa-Nyahkoon L, Sadikova E, Mitchell S, et al. Reducing preconception risks among African American women with conversational agent technology. J Am Board Fam Med. 2015;28(4):441-51.

37. Morse JE, Moos MK. Reproductive life planning: raising the questions. Matern Child Health J. 2018;22(4):439-44.

38. Hipp SL, Chung-Do J, McFarlane E. Systematic review of interventions for reproductive life planning. J Obstet Gynecol Neonatal Nurs. 2019;48(2):131-9.

39. Lang AY, Boyle JA, Fitzgerald GL, Teede H, Mazza D, Moran LJ, et al. Optimizing preconception health in women of reproductive age. Minerva Ginecol. 2018;70(1):99-119.

40. Stephenson J, Vogel C, Hall J, Hutchinson J, Mann S, Duncan H, et al. Preconception health in England: a proposal for annual reporting with core metrics. Lancet. 2019;393(10187):2262-71. 
Table 1: Maternal socio-demographic characteristics and preconception health behaviours by pregnancy planning status

\begin{tabular}{|c|c|c|c|c|}
\hline & $\begin{array}{c}\text { Study } \\
\text { participants } \\
\mathbf{n}=317\end{array}$ & Planned & Unplanned & p-value \\
\hline & n (\%) & n (\%) & n (\%) & \\
\hline Totals & & $236(74.4)$ & $81(25.6)$ & \\
\hline Age [Median (IQR) $30(27,33)$ years] & $\mathrm{n}=284$ & $30(27,33)$ & $29.5(26.3,32.8)$ & 0.10 \\
\hline$<25$ & $36(12.7)$ & $22(61.1)$ & $14(38.9)$ & 0.08 \\
\hline$\geq 25$ & $248(87.3)$ & $186(75.0)$ & $62(25.0)$ & \\
\hline Relationship status & $\mathrm{n}=273$ & & & \\
\hline Married/de facto & $255(93.4)$ & $196(76.9)$ & $59(23.1)$ & $<0.001$ \\
\hline Unmarried* $^{*}$ & $18(6.6)$ & $6(33.3)$ & $12(66.7)$ & \\
\hline Place of birth & $\mathrm{n}=273$ & & & \\
\hline Australia & $157(57.5)$ & $111(70.7)$ & $46(29.3)$ & 0.15 \\
\hline Outside Australia & $116(42.5)$ & $91(78.4)$ & $25(21.6)$ & \\
\hline Previous live birth & $\mathrm{n}=287$ & & & \\
\hline Yes & $148(51.6)$ & $105(70.9)$ & $43(29.1)$ & 0.31 \\
\hline No & $139(48.4)$ & $106(76.3)$ & $33(23.7)$ & \\
\hline Children & $\mathrm{n}=287$ & & & \\
\hline$\leq 2$ & $270(94.1)$ & $203(75.2)$ & $67(24.8)$ & 0.01 \\
\hline$\geq 3$ & $17(5.9)$ & $8(47.1)$ & $9(52.9)$ & \\
\hline Education & $\mathrm{n}=273$ & & & \\
\hline Post-secondary school & $228(83.5)$ & $172(75.4)$ & $56(24.6)$ & 0.22 \\
\hline School only & $45(16.5)$ & $30(66.7)$ & $15(33.3)$ & \\
\hline Employment & $\mathrm{N}=273$ & & & \\
\hline Paid employment & $211(77.3)$ & $159(75.4)$ & $52(24.6)$ & 0.34 \\
\hline Unpaid employment/ unemployed & $62(22.7)$ & $43(69.4)$ & $19(30.6)$ & \\
\hline Public/ private healthcare & $\mathrm{n}=317$ & & & \\
\hline Private & $92(29.0)$ & $79(85.9)$ & $13(14.1)$ & 0.003 \\
\hline Public & $225(71.0)$ & $157(69.8)$ & $68(30.2)$ & \\
\hline
\end{tabular}




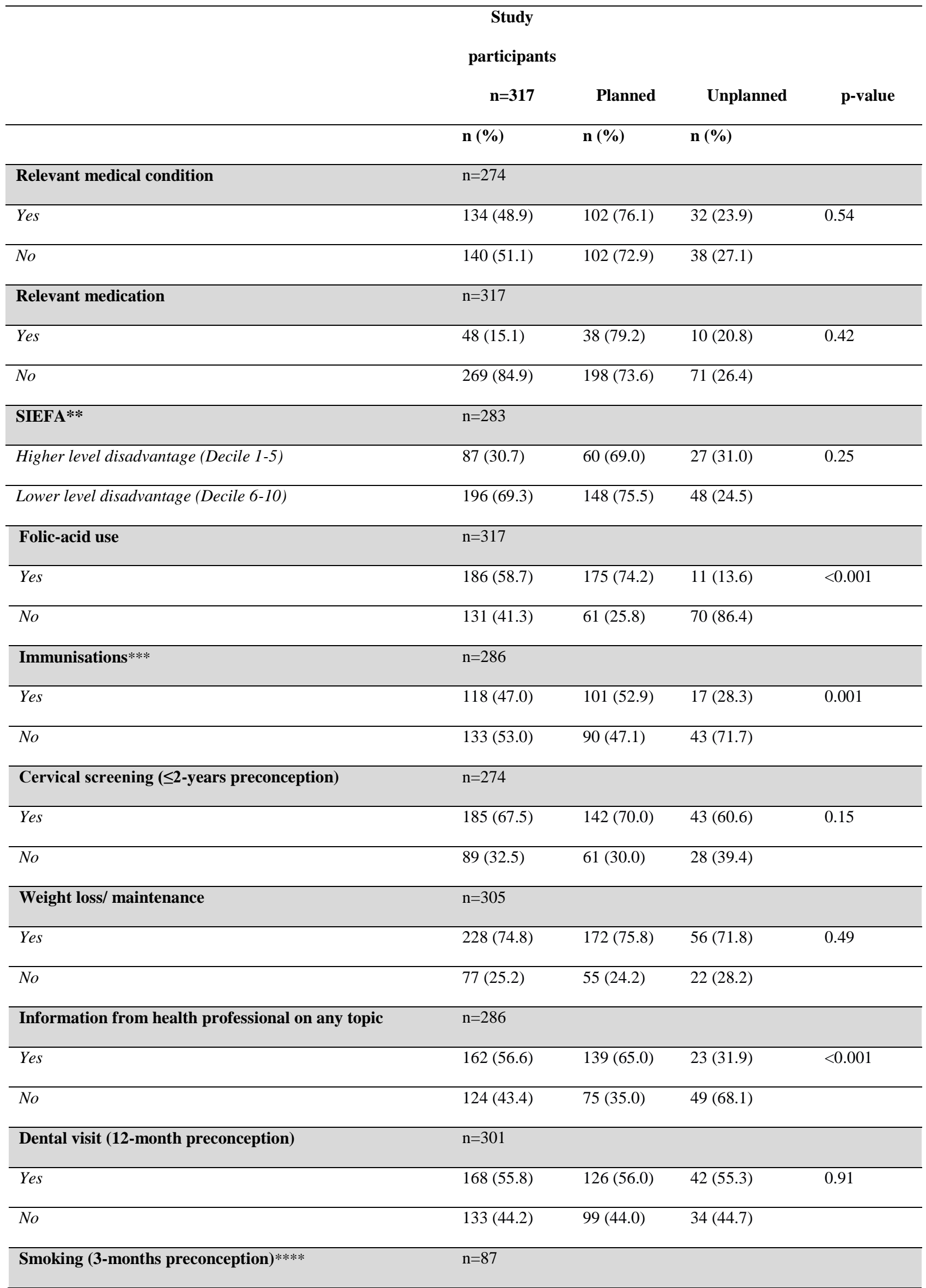




\begin{tabular}{|c|c|c|c|c|}
\hline & $\begin{array}{c}\text { Study } \\
\text { participants } \\
\text { n=317 }\end{array}$ & Planned & Unplanned & p-value \\
\hline & $\mathrm{n}(\%)$ & n (\%) & n (\%) & \\
\hline Yes & $30(34.5)$ & $15(26.3)$ & $15(50.0)$ & 0.03 \\
\hline No/ No, given up & $57(65.5)$ & $42(73.7)$ & $15(50.0)$ & \\
\hline $\begin{array}{l}\text { Drinking alcohol ( } \geq 1 \text { standard drink/week 3-months } \\
\text { preconception) })^{* * * * *}\end{array}$ & $\mathrm{n}=216$ & & & \\
\hline Yes (any amount/ binge) & $139(64.4)$ & $95(60.1)$ & $44(75.9)$ & 0.03 \\
\hline None/ Stopped-trying for pregnancy & 77 (35.6) & $63(39.9)$ & $14(24.1)$ & \\
\hline Illicit drug use & $\mathrm{n}=300$ & & & \\
\hline Yes (ever taken drugs) & $52(17.3)$ & $33(14.7)$ & $19(25.0)$ & 0.04 \\
\hline No & $248(82.7)$ & $191(85.3)$ & $57(75.0)$ & \\
\hline
\end{tabular}

Total $n$ for each variable may vary based on the total number of responses.

*Never married/widow/divorced/separated.

**Socio-economic status was estimated according to participant's postcode, by the deciles in the Australian Socio-economic Indexes for Areas (SEIFA), Index of Relative Socio-economic Disadvantage. ${ }^{20}$ Deciles 1-5 were classified as higher level disadvantage and decile 6-10 as lower level disadvantage.

***Immunisations reviewed included: $\mathrm{n}=109$ MMR and Hepatitis B and/or Chickenpox, $\mathrm{n}=6$ Hepatitis B only, $\mathrm{n}=2 \mathrm{Chickenpox}$ only, $\mathrm{n}=1$ Hepatitis B and Chickenpox only.

**** Only includes women who had ever smoked.

******Only includes women who ever drank alcohol. 
Table 2: Associations between maternal socio-demographic characteristics and pregnancy planning status

\begin{tabular}{|c|c|c|c|c|}
\hline & Univariable & & Multivariable $^{1}$ & \\
\hline Totals & OR $(95 \% \mathrm{CI})$ & p-value & OR $(95 \% \mathrm{CI})$ & p-value \\
\hline $\begin{array}{l}\text { Age [M } \\
(n=284)\end{array}$ & & & & \\
\hline$<25$ & $0.52(0.25-1.09)$ & 0.08 & & \\
\hline
\end{tabular}

\begin{tabular}{lllll}
\hline Relationship status $^{1}(\mathbf{n}=\mathbf{2 7 3})$ & & & \\
& & & & \\
\hline Married/de facto & $6.64(2.39-18.47)$ & $<0.001$ & $5.71(1.92-17.00)$ & 0.002 \\
\hline Unmarried $^{*}$ & Ref & & & \\
\hline
\end{tabular}

\begin{tabular}{lll}
\hline Place of birth $\mathbf{( n = 2 7 3 )}$ & \\
\hline Australia & $0.66(0.38-1.16)$ & 0.15 \\
\hline Outside Australia & Ref & \\
\hline
\end{tabular}

\begin{tabular}{lll}
\hline Previous live birth $(\mathbf{n}=\mathbf{2 8 7})$ & \\
\hline Yes & $0.76(0.45-1.29)$ & 0.31 \\
\hline No & Ref & \\
\hline
\end{tabular}

\begin{tabular}{lllll}
\hline Children $^{1}(\mathbf{n}=\mathbf{2 8 7})$ & & & & \\
\hline$\leq 2$ & $3.41(1.27-9.19)$ & 0.02 & $3.75(1.28-11.05)$ & 0.02 \\
\hline$\geq 3$ & Ref & & \\
\hline Education $(\mathbf{n}=\mathbf{2 7 3})$ & & & \\
\hline Post-secondary school & $1.54(0.77-3.06)$ & 0.22 \\
\hline School only & Ref & \\
\hline
\end{tabular}

\begin{tabular}{lll}
\hline Employment (n=273) & & \\
\hline Paid employment & $1.35(0.72-2.52)$ & 0.35 \\
\hline Unpaid employment/ unemployed & Ref & \\
\hline
\end{tabular}

\begin{tabular}{lllll}
\hline Public/ private healthcare ${ }^{1}(\mathbf{n}=\mathbf{3 1 7})$ & & & & \\
\hline Private & $2.63(1.37-5.05)$ & 0.004 & $2.51(1.08-5.81)$ & 0.03 \\
\hline Public & Ref & & & \\
& & & & \\
\hline Relevant medical condition $(\mathbf{n}=\mathbf{2 7 4})$ & & & \\
\hline Yes & $1.19(0.69-2.05)$ & 0.54 &
\end{tabular}




\begin{tabular}{lcccc}
\hline & Univariable & & Multivariable $^{1}$ & \\
Totals & OR $(95 \%$ CI $)$ & p-value & OR $(95 \%$ CI $)$ & p-value \\
\hline
\end{tabular}

No Ref

\begin{tabular}{lll}
\hline Relevant medication $(\mathbf{n}=\mathbf{3 1 7})$ & & \\
\hline Yes & $1.36(0.65-2.88)$ & 0.42 \\
\hline No & Ref
\end{tabular}

\begin{tabular}{lll}
\hline SIEFA $* *(\mathbf{n}=\mathbf{2 8 3})$ & \\
\hline Higher level disadvantage (Decile 1-5) & $0.72(0.41-1.26)$ & 0.25 \\
\hline
\end{tabular}

Lower level disadvantage (Decile 6-10)

Ref

Total $n$ for each variable may vary based on the total number of responses.

*Never married/widow/divorced/separated.

**Socio-economic status was estimated according to participant's postcode, by the deciles in the Australian Socio-economic Indexes for

Areas (SEIFA), Index of Relative Socio-economic Disadvantage. ${ }^{20}$ Deciles 1-5 were classified as higher level disadvantage and decile 6-10 as lower level disadvantage.

${ }^{1}$ Multivariable analysis was performed for each of the socio-demographic characteristics adjusting for all other variables listed in Table 2. 
Table 3: Associations between health behaviours and pregnancy planning status

\begin{tabular}{|c|c|c|c|c|}
\hline \multirow{2}{*}{ Total } & Univariable & p-value & Multivariable & \multirow[t]{2}{*}{ p-value } \\
\hline & OR $(95 \% \mathrm{CI})$ & & OR $(95 \%$ CI $)$ & \\
\hline \multicolumn{5}{|c|}{ Folic-acid use $^{1}(n=317)$} \\
\hline Yes & $18.26(9.07-36.74)$ & $<0.001$ & $17.13(7.67-38.26)$ & $<0.001$ \\
\hline \multicolumn{5}{|l|}{ No } \\
\hline \multicolumn{5}{|c|}{ Immunisations $* 2(n=286)$} \\
\hline Yes & $2.84(1.51-5.33)$ & 0.001 & $2.09(1.07-4.10)$ & 0.03 \\
\hline \multicolumn{5}{|l|}{ No } \\
\hline \multicolumn{5}{|c|}{ Cervical screening $^{3}$ ( $\leq 2$-years preconception) $(n=274)$} \\
\hline Yes & $1.52(0.86-2.66)$ & 0.15 & & \\
\hline \multicolumn{5}{|l|}{ No } \\
\hline \multicolumn{5}{|c|}{ Weight loss/ maintenance $(n=305)^{4}$} \\
\hline Yes & $1.23(0.69-2.19)$ & 0.49 & & \\
\hline
\end{tabular}

\begin{tabular}{lllll}
\hline Information from health professional on any topic & & & & \\
$(\mathbf{n = 2 8 6})$ & & & & \\
\hline Yes & $3.95(2.23-6.98)$ & $<0.001$ & $3.24(1.75-6.00)$ & $<0.001$ \\
\hline No & & & & \\
\hline
\end{tabular}

\begin{tabular}{|c|c|c|c|c|}
\hline \multicolumn{5}{|c|}{ Dental visit (12-month preconception $)^{6}(\mathrm{n}=301)$} \\
\hline Yes & $1.03(0.61-1.74)$ & 0.91 & & \\
\hline \multicolumn{5}{|l|}{ No } \\
\hline \multicolumn{5}{|c|}{ Smoking (3-months preconception)**7 $(n=87)$} \\
\hline Yes & $0.36(0.14-0.90)$ & 0.03 & $0.44(0.14-1.35)$ & 0.15 \\
\hline
\end{tabular}

No/ No, given up

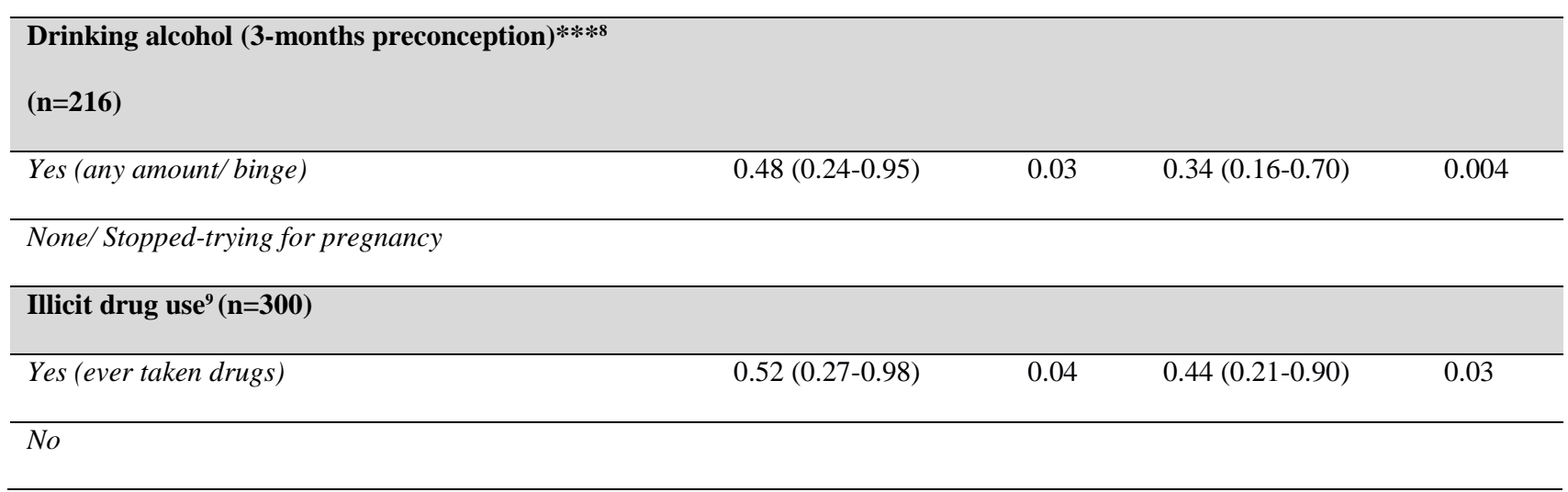


Unplanned pregnancy is the reference group.

Total $n$ for each variable may vary based on the total number of responses.

*Immunisations reviewed included: n=109 MMR and Hepatitis B and/or Chickenpox, n=6 Hepatitis B only, n=2 Chickenpox only, n=1 Hepatitis B and Chickenpox only.

** Only includes women who had ever smoked.

***Only includes women who ever drank alcohol.

${ }^{1}$ Adjustment for pregnancy planning, age, relationship status, previous live birth, education, public/private healthcare, SEIFA, number of children.

${ }^{2}$ Adjustment for pregnancy planning, relationship status, previous live birth, employment, public/private healthcare, number of children.

${ }^{3}$ Adjustment for pregnancy planning, age, country of birth, employment, public/private healthcare.

${ }^{4}$ No significant socio-demographic factors for multivariable adjustment.

${ }^{5}$ Adjustment for pregnancy planning, age, relationship status, country of birth, previous live birth, number of children, education, public/private healthcare.

${ }^{6}$ Adjustment for age, previous live birth, employment, public/private healthcare.

${ }^{7}$ Adjustment for pregnancy planning, age, education, public/private healthcare.

${ }^{8}$ Adjustment for pregnancy planning, previous live birth, public/private healthcare.

${ }^{9}$ Adjustment for pregnancy planning, country of birth, education, relevant medical condition, public/private healthcare. 